\section{Fermi-LAT Observations of the Gamma-Ray Burst GRB 130427A}

M. Ackermann, ${ }^{1}$ M. Ajello, ${ }^{2}$ K. Asano, ${ }^{3}$ W. B. Atwood, ${ }^{4}$ M. Axelsson, ${ }^{5,6,7}$ L. Baldini, ${ }^{8}$ ]. Ballet, ${ }^{9}$ G. Barbiellini, ${ }^{10,11}$ M. G. Baring ${ }^{12}$ D. Bastieri, ${ }^{13,14}$ K. Bechtol, ${ }^{15}$ R. Bellazzini, ${ }^{16} \mathrm{E}$. Bissaldi, ${ }^{17}$ E. Bonamente, ${ }^{18,19}$ ]. Bregeon, ${ }^{16}$ M. Brigida, ${ }^{20,21}$ P. Bruel, $^{22}$ R. Buehler, $^{1}$ J. Michael Burgess, $^{23}$ S. Buson, ${ }^{13,14}$ G. A. Caliandro, ${ }^{24}$ R. A. Cameron, ${ }^{15}$ P. A. Caraveo, ${ }^{25}$ C. Cecchi, ${ }^{18,19}$ V. Chaplin, ${ }^{23}$ E. Charles, ${ }^{15}$ A. Chekhtman, ${ }^{26}$ C. C. Cheung, ${ }^{27}$ J. Chiang, ${ }^{15 *}$ G. Chiaro, ${ }^{14}$ S. Ciprini, ${ }^{28,29}$ R. Claus, ${ }^{15}$ W. Cleveland, ${ }^{30}$ ]. Cohen-Tanugi, ${ }^{31}$ A. Collazzi, ${ }^{32}$ L. R. Cominsky, ${ }^{33}$ V. Connaughton, ${ }^{23}$ J. Conrad, ${ }^{34,6,35,36}$ S. Cutini, ${ }^{28,29}$ F. D'Ammando, ${ }^{37}$ A. de Angelis, ${ }^{38}$ M. DeKlotz, ${ }^{39}$ F. de Palma, ${ }^{20,21}$ C. D. Dermer, ${ }^{27 *}$ R. Desiante, ${ }^{10}$ A. Diekmann, ${ }^{40}$ L. Di Venere, ${ }^{15}$ P. S. Drell, ${ }^{15}$ A. Drlica-Wagner, ${ }^{15}$ C. Favuzzi, ${ }^{20,21}$ S. J. Fegan, ${ }^{22}$ E. C. Ferrara, ${ }^{41}$ ]. Finke, ${ }^{27}$ G. Fitzpatrick, ${ }^{42}$ W. B. Focke, ${ }^{15}$

A. Franckowiak, ${ }^{15}$ Y. Fukazawa, ${ }^{43}$ S. Funk, ${ }^{15}$ P. Fusco, ${ }^{20,21}$ F. Gargano, ${ }^{21}$ N. Gehrels, ${ }^{41}$ S. Germani, ${ }^{18,19}$ M. Gibby, ${ }^{40}$ N. Giglietto, ${ }^{20,21}$ M. Giles, ${ }^{40}$ F. Giordano, ${ }^{20,21}$ M. Giroletti, ${ }^{37}$ G. Godfrey, ${ }^{15}$ ]. Granot, ${ }^{44}$ I. A. Grenier, ${ }^{9}$ ]. E. Grove, ${ }^{27}$ D. Gruber, ${ }^{45}$ S. Guiriec, ${ }^{41,32}$ D. Hadasch, ${ }^{24}$ Y. Hanabata, ${ }^{43}$ A. K. Harding, ${ }^{41}$ M. Hayashida, ${ }^{15,46}$ E. Hays, ${ }^{41}$ D. Horan, ${ }^{22}$ R. E. Hughes, ${ }^{47}$ Y. Inoue, ${ }^{15}$ T. Jogler, $^{15}$ G. Jóhannesson, ${ }^{48}$ W. N. Johnson, ${ }^{27}$ T. Kawano, ${ }^{43}$ J. Knödlseder, ${ }^{49,50}$ D. Kocevski, ${ }^{15}$ M. Kuss, ${ }^{16}$ J. Lande, ${ }^{15}$ S. Larsson, ${ }^{34,6,5}$ L. Latronico, ${ }^{51}$ F. Longo, ${ }^{10,11}$ F. Loparco, ${ }^{20,21}$ M. N. Lovellette, ${ }^{27}$ P. Lubrano, ${ }^{18,19}$ M. Mayer, ${ }^{1}$ M. N. Mazziotta, ${ }^{21}$ ]. E. McEnery, ${ }^{41,52}$ P. F. Michelson, ${ }^{15}$ T. Mizuno, ${ }^{53}$ A. A. Moiseev, ${ }^{54,52}$ M. E. Monzani, ${ }^{15}$ E. Moretti, ${ }^{7,6}$ A. Morselli, ${ }^{55}$ I. V. Moskalenko, ${ }^{15}$ S. Murgia, ${ }^{15}$ R. Nemmen, ${ }^{41}$ E. Nuss, ${ }^{31}$ M. Ohno, ${ }^{43}$ T. Ohsugi, ${ }^{53}$ A. Okumura, ${ }^{15,56}$ N. Omodei, ${ }^{15 *}$ M. Orienti, ${ }^{37}$ D. Paneque, ${ }^{57,15}$ V. Pelassa, ${ }^{23}$ J. S. Perkins, ${ }^{41,58,54}$ M. Pesce-Rollins, ${ }^{16}$ V. Petrosian, ${ }^{15}$ F. Piron, ${ }^{31}$ G. Pivato, ${ }^{14}$ T. A. Porter, ${ }^{15}$ J. L. Racusin, ${ }^{41}$ S. Rainò ${ }^{20,21}$ R. Rando, ${ }^{13,14}$ M. Razzano, ${ }^{16,4}$ S. Razzaque, ${ }^{59}$ A. Reimer, ${ }^{60,15}$ O. Reimer, ${ }^{60,15}$ S. Ritz, ${ }^{4}$ M. Roth, ${ }^{61}$ F. Ryde, ${ }^{7,6}$ A. Sartori, ${ }^{25}$ P. M. Saz Parkinson, ${ }^{4}$ ]. D. Scargle, ${ }^{62}$ A. Schulz, ${ }^{1}$ C. Sgrò, ${ }^{16}$ E. J. Siskind, ${ }^{63}$ E. Sonbas, ${ }^{41,64,30}$ G. Spandre, ${ }^{16}$ P. Spinelli, ${ }^{20,21}$ H. Tajima, ${ }^{15,56}$ H. Takahashi, ${ }^{43}$ J. G. Thayer, ${ }_{15}^{15}$ J. B. Thayer, ${ }^{15}$ D. J. Thompson, ${ }^{41}$ L. Tibaldo, $^{15}$ M. Tinivella, $^{16}$ D. F. Torres, ${ }^{24,65}$ G. Tosti, ${ }^{18,19}$ E. Troja, ${ }^{41,52}$ T. L. Usher, ${ }^{15}$ ]. Vandenbroucke, ${ }^{15}$ V. Vasileiou, ${ }^{31}$ G. Vianello, ${ }^{15,66 *}$ V. Vitale, ${ }^{55,67}$ B. L. Winer, ${ }^{47}$ K. S. Wood, ${ }^{27}$ R. Yamazaki, ${ }^{68}$ G. Younes, $^{30,69}$ H.-F. Yu, ${ }^{45}$ S. J. Zhu, ${ }^{52 *}$ P. N. Bhat, ${ }^{23}$ M. S. Briggs, ${ }^{23}$ D. Byrne, ${ }^{42}$ S. Foley, ${ }^{42,45}$ A. Goldstein, ${ }^{23}$ P. Jenke, ${ }^{23}$ R. M. Kippen, ${ }^{70}$ C. Kouveliotou, ${ }^{69}$ S. McBreen, ${ }^{42,45}$ C. Meegan, ${ }^{23}$ W. S. Paciesas, ${ }^{30}$ R. Preece, ${ }^{23}$ A. Rau $^{45}$ D. Tierney, ${ }^{42}$ A. J. van der Horst, ${ }^{71}$ A. von Kienlin, ${ }^{45}$ C. Wilson-Hodge, ${ }^{69}$ S. Xiong, ${ }^{23 *}$ G. Cusumano, ${ }^{72}$ V. La Parola, ${ }^{72}$ J. R. Cummings ${ }^{41,73}$

The observations of the exceptionally bright gamma-ray burst (GRB) 130427A by the Large Area Telescope aboard the Fermi Gamma-ray Space Telescope provide constraints on the nature of these unique astrophysical sources. GRB 130427A had the largest fluence, highest-energy photon ( $95 \mathrm{GeV}$ ), longest $\gamma$-ray duration (20 hours), and one of the largest isotropic energy releases ever observed from a GRB. Temporal and spectral analyses of GRB 130427A challenge the widely accepted model that the nonthermal high-energy emission in the afterglow phase of GRBs is synchrotron emission radiated by electrons accelerated at an external shock.

$\mathrm{G}$ amma-ray bursts (GRBs) are thought to originate from collapsing massive stars or merging compact objects (such as neutron stars or black holes), and are associated with the formation of black holes in distant galaxies. GRB 130427A was detected by both the Large Area Telescope (LAT) and the Gamma-ray Burst Monitor (GBM) aboard the Fermi Gamma-ray Space Telescope. The LAT is a pair-conversion telescope that observes photons from $20 \mathrm{MeV}$ to $>300 \mathrm{GeV}$ with a 2.4 -steradian field of view (1). The GBM consists of 12 sodium iodide (NaI, $8 \mathrm{keV}$ to $1 \mathrm{MeV}$ ) detectors and two bismuth germanate (BGO, $200 \mathrm{keV}$ to $40 \mathrm{MeV}$ ) detectors, positioned around the spacecraft to view the entire unocculted sky (2).

In the standard model of GRBs, the blast wave that produces the initial, bright prompt emission later collides with the external material surrounding the GRB (the circumburst medium) and cre-
Gamma Ray Observatory more than 90 min after GRB 940217 began (5).

\section{Observations}

At 07:47:06.42 UTC on 27 April $2013\left(T_{0}\right)$, while Fermi was in the regular survey mode, the GBM triggered on GRB 130427A. The burst was sufficiently hard and intense to initiate an autonomous repoint request ( 6 ) - a spacecraft slewing maneuver that keeps the burst within the LAT field of view for 2.5 hours, barring Earth occultation. At the time of the GBM trigger, the Swift Burst Alert Telescope (BAT) was slewing between two planned targets; the BAT triggered on the ongoing burst at 07:47:57.51 UTC immediately after completing the slew (7), 51.1 s after the GBM trigger. The CARMA millimeter-wave observatory localized this burst to R.A. $=173.1367^{\circ}$, Dec. $=27.6989^{\circ}(\mathrm{J} 2000)$ with an uncertainty of $0.4 \operatorname{arcsec}(8)$. The Rapid Telescopes for Optical Response (RAPTOR) detected bright optical emission from the GRB, peaking at a red-band magnitude of $R=7.03 \pm 0.03$ around the GBM trigger time before fading to $R \approx 10$ about $80 \mathrm{~s}$ later $(9)$. The Gemini-North observatory reported a redshift of $z=0.34$ (4), and an underlying supernova has been detected (10). A total of 58 observatories have reported observations of this burst as of September 2013.

At the time of the GBM trigger, the GRB was $47.3^{\circ}$ from the LAT boresight, well within the LAT field of view. The autonomous repoint request brought the burst to $20.1^{\circ}$ from the LAT boresight, based on the position calculated by the GBM flight software. It remained in the LAT field of view for $715 \mathrm{~s}$ until it became occulted by Earth, reemerging from Earth occultation at $T_{0}+$ $3135 \mathrm{~s}$. Within the first $\sim 80 \mathrm{ks}$ after the trigger, the LAT detected more than 500 photons with energies greater than $100 \mathrm{MeV}$ associated with the GRB; the previous record holder was GRB 090902B, with $\sim 200$ photons (11). In addition, the LAT detected 15 photons with energies greater than $10 \mathrm{GeV}$ (versus only 3 photons for GRB 090902B). Using the LAT Low Energy (LLE) event selection (12), which considerably increases the LAT effective collecting area to lower-energy $\gamma$-rays down to $10 \mathrm{MeV}$ (with adequate energy reconstruction down to $30 \mathrm{MeV})(13,14)$, thousands of counts above background were detected between $T_{0}$ and $T_{0}+100 \mathrm{~s}$.

\section{Temporal Characteristics}

The temporal profile of the emission from GRB 130427A varies strongly with energy from $10 \mathrm{keV}$ to $\sim 100 \mathrm{GeV}$ (Figs. 1 and 2). The GBM light curves consist of an initial peak lasting for a few seconds, a much brighter multipeaked emission episode lasting $\sim 10 \mathrm{~s}$, and a dim, broad peak at $T_{0}+120 \mathrm{~s}$, which fades to an undetectable level after $\sim 300 \mathrm{~s}$ [also seen in the Swift light curve (7)].

The triggering pulse observed in the LLE $(>10 \mathrm{MeV})$ light curve is more sharply peaked than the NaI- and BGO-detected emission at $T_{0}$. 
The LLE light curve between $T_{0}+4 \mathrm{~s}$ and $T_{0}+$ $12 \mathrm{~s}$ exhibits a multipeaked structure. Some of these peaks have counterparts in the GBM energy range, although the emission episodes are not perfectly correlated (e.g., the sharp spike in the LLE light curve at $T_{0}+9.5 \mathrm{~s}$ is not relatively bright in the GBM light curves) because of the spectral evolution with energy.

The LAT-detected emission, however, does not appear to be temporally correlated with either the LLE or GBM emission beyond the initial spike at $T_{0}$. Photons with energies greater than $1 \mathrm{GeV}$ are first observed $\sim 10 \mathrm{~s}$ after $T_{0}$, after the brightest GBM emission has ended, consistent with a delayed onset of the high-energy emission (13). The delayed onset is not caused by a progressively increasing LAT acceptance due to slewing, because the slew started at $T_{0}+33 \mathrm{~s}$. Instead, it reflects the true evolution of the GRB emission.

GRB spectra are generally well described by phenomenological models such as the Band function (15) or the smoothly broken power law [SBPL (10)]. For the brightest LAT bursts, the onset of the $\mathrm{GeV}$ emission is delayed with respect to the $\mathrm{keV}-\mathrm{MeV}$ emission and can be fit by an additional power-law component (13). This additional component usually becomes significant while the $\mathrm{keV}-\mathrm{MeV}$ emission is still bright.

For GRB 130427A, however, the extra powerlaw component becomes significant only after the GBM-detected emission has faded (Fig. 3). During the initial peak $\left(T_{0}-0.1 \mathrm{~s}\right.$ to $\left.T_{0}+4.5 \mathrm{~s}\right)$, there are only a few LAT-detected photons, and the emission is well fit by an SBPL. For the brightest part of the burst $\left(T_{0}+4.5 \mathrm{~s}\right.$ to $T_{0}+$ $11.5 \mathrm{~s}$ ), we did not use the GBM-detected emission because of the substantial systematic effects caused by extremely high flux (17); however, there are no photons with energies greater than $1 \mathrm{GeV}$ in this time interval, and the energy spectrum above $30 \mathrm{MeV}$ is well described by a single power law without a break (Fig. 3) (note that the LAT did not suffer from any pile-up issues). Photons with energies greater than $1 \mathrm{GeV}$ are detected in the last time interval $\left(T_{0}+11.5 \mathrm{~s}\right.$ to $T_{0}+33.0 \mathrm{~s}$ ), including a $73-\mathrm{GeV}$ photon at $T_{0}+$ $19 \mathrm{~s}$. Unlike other bright LAT bursts, the LATdetected emission from GRB 130427A appears to be temporally distinct from the GBM-detected emission, which suggests that the $\mathrm{GeV}$ and $\mathrm{keV}$ $\mathrm{MeV}$ photons arise from different emission regions or mechanisms.

\section{Temporally Extended High-Energy Emission}

To characterize the temporally extended highenergy emission, we performed an unbinned maximum likelihood analysis of the LAT data for $E>100 \mathrm{MeV}$. We modeled the LAT photon spectrum as a power law with a spectral index $\alpha$ [i.e., the spectrum $N(E) \propto E^{\alpha}$ ]. We found evidence of spectral evolution during the high- energy emission. In contrast to another study (18) that used longer time intervals in the spectral fits, we found that the LAT $(E>100 \mathrm{MeV})$ spectrum of the GRB is well described by a power law at all times, but with a varying spectral index (17).

During the first pulse around $T_{0}$, emission at $>100 \mathrm{MeV}$ is faint and soft; the pulse contains only a few photons, and their energies are all $<1 \mathrm{GeV}$ (Fig. 2). This is followed by a period during which there is no significant emission at $>100 \mathrm{MeV}$, while the GBM emission is at its brightest. Starting around $T_{0}+5 \mathrm{~s}$, emission at $>100 \mathrm{MeV}$ is detectable again but remains dim until $\sim T_{0}+12 \mathrm{~s}$. The spectral index fluctuates between $\alpha \sim-2.5$ and $\alpha \sim-1.7$. At late times (longer than $T_{0}+300 \mathrm{~s}$ ), we measured typical spectral indices of $\alpha \sim-2$, consistent with the indices of other LAT bursts (13). During the time intervals with the hardest spectra, the LAT observed the highest-energy photons - such as the $73-\mathrm{GeV}$ photon at $T_{0}+19 \mathrm{~s}$ and the recordbreaking $95-\mathrm{GeV}$ photon at $T_{0}+244 \mathrm{~s}$ - that severely restrict the possible mechanisms that could generate the high-energy afterglow emission (table S2).

The temporally extended photon flux light curve is better fit by a broken power law than by a single power law. We found a break after a few hundred seconds, with the temporal index steepening from $-0.85 \pm 0.08$ to $-1.35 \pm 0.08$
${ }^{1}$ Deutsches Elektronen Synchrotron DESY, D-15738 Zeuthen, Germany. ${ }^{2}$ Space Sciences Laboratory, University of California, Berkeley, CA 94720, USA. ${ }^{3}$ Institute for Cosmic Ray Research, University of Tokyo, 5-1-5 Kashiwa-no-Ha, Kashiwa City, Chiba 277-8582, Japan. ${ }^{4}$ Santa Cruz Institute for Particle Physics, Department of Physics, and Department of Astronomy and Astrophysics, University of California, Santa Cruz, CA 95064, USA. ${ }^{5}$ Department of Astronomy, Stockholm University, SE-106 91 Stockholm, Sweden. ${ }^{6}$ Oskar Klein Centre for Cosmoparticle Physics, AlbaNova, SE-106 91 Stockholm, Sweden. ${ }^{7}$ Department of Physics, Royal Institute of Technology (KTH), AlbaNova, SE-106 91 Stockholm, Sweden. ${ }^{8}$ Università di Pisa and Istituto Nazionale di Fisica Nucleare, Sezione di Pisa I-56127 Pisa, Italy. 'Laboratoire AIM, CEA-IRFU/CNRS/Université Paris Diderot, Service d'Astrophysique, CEA Saclay, 91191 Gif sur Yvette, France. ${ }^{10}$ Istituto Nazionale di Fisica Nucleare, Sezione di Trieste, 1-34127 Trieste, Italy. ${ }^{11}$ Dipartimento di Fisica, Università di Trieste, I-34127 Trieste, Italy. ${ }^{12}$ Department of Physics and Astronomy, Rice University, Houston, TX 77251, USA. ${ }^{13}$ Istituto Nazionale di Fisica Nucleare, Sezione di Padova, I-35131 Padova, Italy. ${ }^{14}$ Dipartimento di Fisica e Astronomia "G. Galilei," Università di Padova, I-35131 Padova, Italy. ${ }^{15}$ W. W. Hansen Experimental Physics Laboratory, Kavli Institute for Particle Astrophysics and Cosmology, Department of Physics and SLAC National Accelerator Laboratory, Stanford University, Stanford, CA 94305, USA. ${ }^{16}$ Istituto Nazionale di Fisica Nucleare, Sezione di Pisa, I-56127 Pisa, Italy. ${ }^{17}$ Istituto Nazionale di Fisica Nucleare, Sezione di Trieste, and Università di Trieste, I-34127 Trieste, Italy. ${ }^{18}$ Istituto Nazionale di Fisica Nucleare, Sezione di Perugia, I-06123 Perugia, Italy. ${ }^{19}$ Dipartimento di Fisica, Università degli Studi di Perugia, I-06123 Perugia, Italy. ${ }^{20}$ Dipartimento di Fisica "M. Merlin" dell'Università e del Politecnico di Bari, I-70126 Bari, Italy. ${ }^{21}$ Istituto Nazionale di Fisica Nucleare, Sezione di Bari, 70126 Bari, Italy. ${ }^{22}$ Laboratoire Leprince-Ringuet, École Polytechnique, CNRS/IN2P3, Palaiseau, France. ${ }^{23}$ Center for Space Plasma and Aeronomic Research, University of Alabama, Huntsville, AL 35899, USA. ${ }^{24}$ Institut de Ciències de I'Espai (IEEE-CSIC), Campus UAB, 08193 Barcelona, Spain. ${ }^{25}$ INAF-Istituto di Astrofisica Spaziale e Fisica Cosmica, I-20133 Milano, Italy.
${ }^{26}$ Center for Earth Observing and Space Research, College of Science, George Mason University, Fairfax, VA 22030, USA; resident at Naval Research Laboratory, Washington, DC 20375, USA. ${ }^{27}$ Space Science Division, Naval Research Laboratory, Washington, DC 20375, USA. ${ }^{28}$ Agenzia Spaziale Italiana Science Data Center, I-00044 Frascati (Roma), Italy. ${ }^{29}$ Istituto Nazionale di Astrofisica-Osservatorio Astronomico di Roma, I-00040 Monte Porzio Catone (Roma), Italy. ${ }^{30}$ Universities Space Research Association, Columbia, MD 21044, USA. ${ }^{31}$ Laboratoire Univers et Particules de Montpellier, Université Montpellier 2, CNRS/IN2P3, Montpellier, France. ${ }^{32}$ NASA Postdoctoral Program Fellow, USA. ${ }^{33}$ Department of Physics and Astronomy, Sonoma State University, Rohnert Park, CA 94928, USA. ${ }^{34}$ Department of Physics, Stockholm University, AlbaNova, SE-106 91 Stockholm, Sweden. ${ }^{35}$ Royal Swedish Academy of Sciences Research Fellow, funded by a grant from the K. A. Wallenberg Foundation. ${ }^{36}$ Royal Swedish Academy of Sciences, Box 50005, SE-104 05 Stockholm, Sweden. ${ }^{37}$ INAF Istituto di Radioastronomia, 40129 Bologna, Italy. ${ }^{38}$ Dipartimento di Fisica, Università di Udine, and Istituto Nazionale di Fisica Nucleare, Sezione di Trieste, Gruppo Collegato di Udine, I-33100 Udine, Italy. ${ }^{39}$ Stellar Solutions Inc., 250 Cambridge Avenue, Suite 204, Palo Alto, CA 94306, USA. ${ }^{40}$ acobs Technology, Huntsville, AL 35806, USA. ${ }^{41}$ NASA Goddard Space Flight Center, Greenbelt, MD 20771, USA. ${ }^{42}$ University College Dublin, Belfield, Dublin 4, Ireland. ${ }^{43}$ Department of Physical Sciences, Hiroshima University, Higashi-Hiroshima, Hiroshima 739-8526, Japan. ${ }^{44}$ Department of Natural Sciences, Open University of Israel, Ra'anana 43537, Israel. ${ }^{45}$ Max-Planck-Institut für Extraterrestrische Physik, 85748 Garching, Germany. ${ }^{46}$ Department of Astronomy, Graduate School of Science, Kyoto University, Sakyo-ku, Kyoto 606-8502, Japan. ${ }^{47}$ Department of Physics, Center for Cosmology and Astro-Particle Physics, Ohio State University, Columbus, OH 43210, USA. ${ }^{48}$ Science Institute, University of Iceland, IS-107 Reykjavik, Iceland. ${ }^{49}$ CNRS, IRAP, F-31028 Toulouse Cedex 4, France. ${ }^{50}$ GAHEC, Université de Toulouse, UPS-OMP, IRAP, Toulouse, France. ${ }^{51}$ Istituto Nazionale di Fisica Nucleare, Sezione di Torino, I-10125 Torino, Italy. ${ }^{52}$ Department of Physics and Department of Astronomy,
University of Maryland, College Park, MD 20742, USA. ${ }^{53}$ Hiroshima Astrophysical Science Center, Hiroshima University, Higashi-Hiroshima, Hiroshima 739-8526, Japan. ${ }^{54} \mathrm{Center}$ for Research and Exploration in Space Science and Technology (CRESST) and NASA Goddard Space Flight Center, Greenbelt, MD 20771, USA. ${ }^{55}$ Istituto Nazionale di Fisica Nucleare, Sezione di Roma "Tor Vergata," I-00133 Roma, Italy. ${ }^{56}$ Solar-Terrestrial Environment Laboratory, Nagoya University, Nagoya 464-8601, Japan. ${ }^{57}$ Max-Planck-Institut für Physik, D-80805 München, Germany. ${ }^{58}$ Department of Physics and Center for Space Sciences and Technology, University of Maryland Baltimore County, Baltimore, MD 21250, USA. ${ }^{59}$ Department of Physics, University of Johannesburg, Auckland Park 2006, South Africa. ${ }^{60}$ Institut für Astround Teilchenphysik and Institut für Theoretische Physik, Leopold-Franzens-Universität Innsbruck, A-6020 Innsbruck, Austria. ${ }^{61}$ Department of Physics, University of Washington, Seattle, WA 98195, USA. ${ }^{62}$ Space Sciences Division, NASA Ames Research Center, Moffett Field, CA 94035, USA. ${ }^{63}$ NYCB Real-Time Computing Inc., Lattingtown, NY 11560, USA. ${ }^{64}$ Adyaman University, 02040 Adyaman, Turkey. ${ }^{65}$ Institució Catalana de Recerca i Estudis Avançats (ICREA), Barcelona, Spain. ${ }^{66}$ Consorzio Interuniversitario per la Fisica Spaziale (CIFS), I-10133 Torino, Italy. ${ }^{67}$ Dipartimento di Fisica, Università di Roma "Tor Vergata," I-00133 Roma, Italy. ${ }^{68}$ Department of Physics and Mathematics, Aoyama Gakuin University, Sagamihara, Kanagawa 252-5258, Japan. ${ }^{69}$ NASA Marshall Space Flight Center, Huntsville, AL 35812, USA. ${ }^{70}$ Los Alamos National Laboratory, Los Alamos, NM 87545, USA.

${ }^{71}$ Astronomical Institute Änton Pannekoek, University of Amsterdam, 1090 GE Amsterdam, Netherlands. ${ }^{72}$ INAFIstituto di Astrofisica Spaziale e Fisica Cosmica, Via U. La Malfa 153, I-90146 Palermo, Italy. ${ }^{73}$ Center for Research and Exploration in Space Science \& Technology, University of Maryland, Baltimore County, Baltimore, MD 21250, USA.

*Corresponding author. E-mail: sjzhu@umd.edu (S.J.Z.); jchiang@slac.stanford.edu (J.C.); charles.dermer@nrl. navy.mil (C.D.D.); nicola.omodei@stanford.edu (N.O.); giacomov@slac.stanford.edu (G.V.); shaolin.xiong@uah.edu (S.X.) 
$\left(\chi^{2} / \mathrm{df}=36 / 19\right.$ for a single power law, 16/17 for a broken power law). In contrast, a break is not statistically preferred in the energy flux light curve $\left(\chi^{2} / \mathrm{df}=14 / 18\right.$ for a single power law, $13 / 17$ for a broken power law), probably because of the larger statistical uncertainties. For a single power-law fit to the energy flux light curve, we found a temporal index of $-1.17 \pm 0.06$, consistent with other LAT bursts (13).

The GBM and Swift energy flux light curves are also shown in Fig. 2. The Swift X-Ray Telescope (XRT) began observing the burst at $T_{0}+$ $190 \mathrm{~s}$; the reported XRT + BAT (0.3 to $10 \mathrm{keV})$ light curve is a combination of XRT data and BAT-detected emission (15 to $150 \mathrm{keV}$ ) extrapolated down into the energy range of the XRT. The $\mathrm{XRT}+\mathrm{BAT}$ light curve shows the unabsorbed flux in the range 0.3 to $10 \mathrm{keV}$ (7). During the initial part of the burst, the GBM (10 keV to $10 \mathrm{MeV}$ ) light curve peaks earlier than both the $\mathrm{XRT}+\mathrm{BAT}(0.3$ to $10 \mathrm{keV})$ and LAT $(>100 \mathrm{MeV})$ light curves. The GBM light curve peaks again at $\sim T_{0}+120 \mathrm{~s}$ [see also (7)], whereas the LAT light curve shows a sharp and hard peak at $T_{0}+200 \mathrm{~s}$. The BAT + XRT light curve peaks again as well at the same time as the LAT light curve, but the peak is much broader.

\section{Interpretation}

The energetics of GRB 130427A place it among the brightest LAT bursts. For GRB 130427A, the fluence at $10 \mathrm{keV}$ to $20 \mathrm{MeV}$ measured with the GBM in the $400 \mathrm{~s}$ following $T_{0}$ is $\sim 4.2 \times$ $10^{-3} \mathrm{ergs}^{-2}$. The issue with pulse pile-up and the uncertainties in the calibration of the GBM detectors contribute to a systematic error that we estimate to be less than $20 \%$; the statistical uncertainty $\left[0.01 \times 10^{-3} \mathrm{ergs} \mathrm{cm}^{-2}\right]$ is negligible with respect to the systematic one (17). The fluence at $>100 \mathrm{MeV}$ measured with the LAT in the $100 \mathrm{ks}$ following $T_{0}$ is $7( \pm 1) \times 10^{-4} \mathrm{ergs} \mathrm{cm}{ }^{-2}$. The total LAT fluence is therefore $\sim 20 \%$ of the GBM flu- ence, similar to other bright LAT GRBs $(13,19)$. For a total fluence at $10 \mathrm{keV}$ to $100 \mathrm{GeV}$ of $4.9 \times$ $10^{-3} \mathrm{ergs} \mathrm{cm}^{-2}$, the total apparent isotropic $\gamma$-ray energy (i.e., the total energy release if there were no beaming) is $E_{\gamma \text {,iso }}=1.40 \times 10^{54} \mathrm{ergs}$, using a flat $\Lambda \mathrm{CDM}$ cosmology with reduced Hubble constant $h=0.71$ and dark energy density $\Omega_{\Lambda}=$ 0.73 ; this value implies a luminosity distance of $1.8 \mathrm{Gpc}$ for $z=0.34$. This value of $E_{\gamma, \text { iso }}$ is only slightly less than the values for other bright LAT hyperenergetic events, which include GRB 080916C, GRB 090902B, and GRB 090926A (19).

The emission region must be transparent against absorption by photon-photon pair production, which has a significant effect at the energies of the LAT-detected emission. Viable models of GRBs therefore require highly relativistic, jetted plasma outflows with bulk jet Lorentz factors $\Gamma$ greater than $\sim 100(20)$. The $73-\mathrm{GeV}$ photon at $T_{0}+19 \mathrm{~s}$ (table S2) provides the most stringent limit on $\Gamma$. Assuming that the variability time scale re-

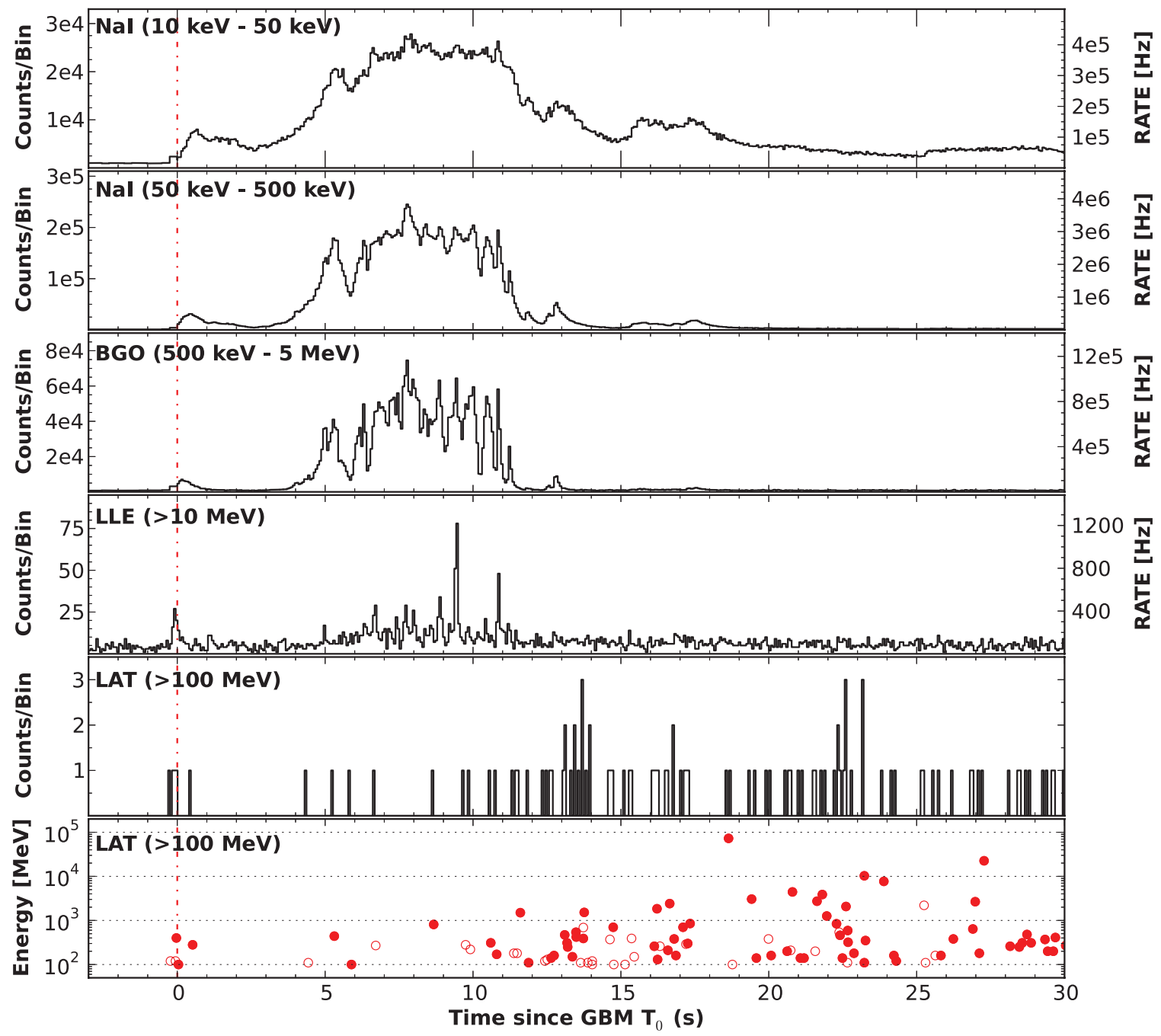

Fig. 1. Light curves for the Fermi-GBM and LAT detectors during the brightest part of the emission in $0.064-\mathrm{s}$ bins, divided into five energy ranges. The $\mathrm{Nal}$ and $\mathrm{BGO}$ light curves were created from a type of GBM data (continuous time or CTIME) that does not suffer from saturation effects induced by the extreme brightness of this GRB (17); for these light curves, we used Nal detectors 6, 9, and 10, and $\mathrm{BGO}$ detector 1 . The open circles in the bottom panel represent the individual LAT "transient" class photons and their energies; the solid circles indicate photons with a $>0.9$ probability of being associated with this burst (17). 
flects the size of the emitting region, and that the $\mathrm{MeV}$ and $\mathrm{GeV}$ emissions around the time of the $73-\mathrm{GeV}$ photon at $T_{0}+19 \mathrm{~s}$ are cospatial, the requirement that the optical depth due to $\gamma \gamma$ opacity be less than 1 then implies that the minimum bulk Lorentz factor is $\Gamma_{\min }=455_{-13}^{+16}$. Here, a SBPL fit to the GBM spectrum in the interval 11.5 to $33.0 \mathrm{~s}$ (table $\mathrm{S} 1$ ) and a minimum variability time scale of $0.04 \pm 0.01 \mathrm{~s}$ are used (17). The cospatial as- sumption is, however, questionable given the different time histories in the $\mathrm{MeV}$ and $\mathrm{GeV}$ emission. Moreover, values of $\Gamma_{\min }$ that are smaller by a factor of 2 to 3 can be realized for models with time-dependent $\gamma$-ray opacity in a thin-shell model (21).

The delayed onset of the LAT-detected emission with respect to the GBM-detected emission is an important clue to the nature of GRBs (13). For
GRB 130427A, the LAT-detected emission becomes harder and more intense after the GBMdetected emission has faded (Fig. 3). This suggests that the $\mathrm{GeV}$ emission is produced later than the $\mathrm{keV}-\mathrm{MeV}$ emission and in a different region. In particular, if the keV-MeV emission comes from interactions within the outflow itself, the $\mathrm{GeV}$ emission arises from the outflow's interactions with the circumburst medium.

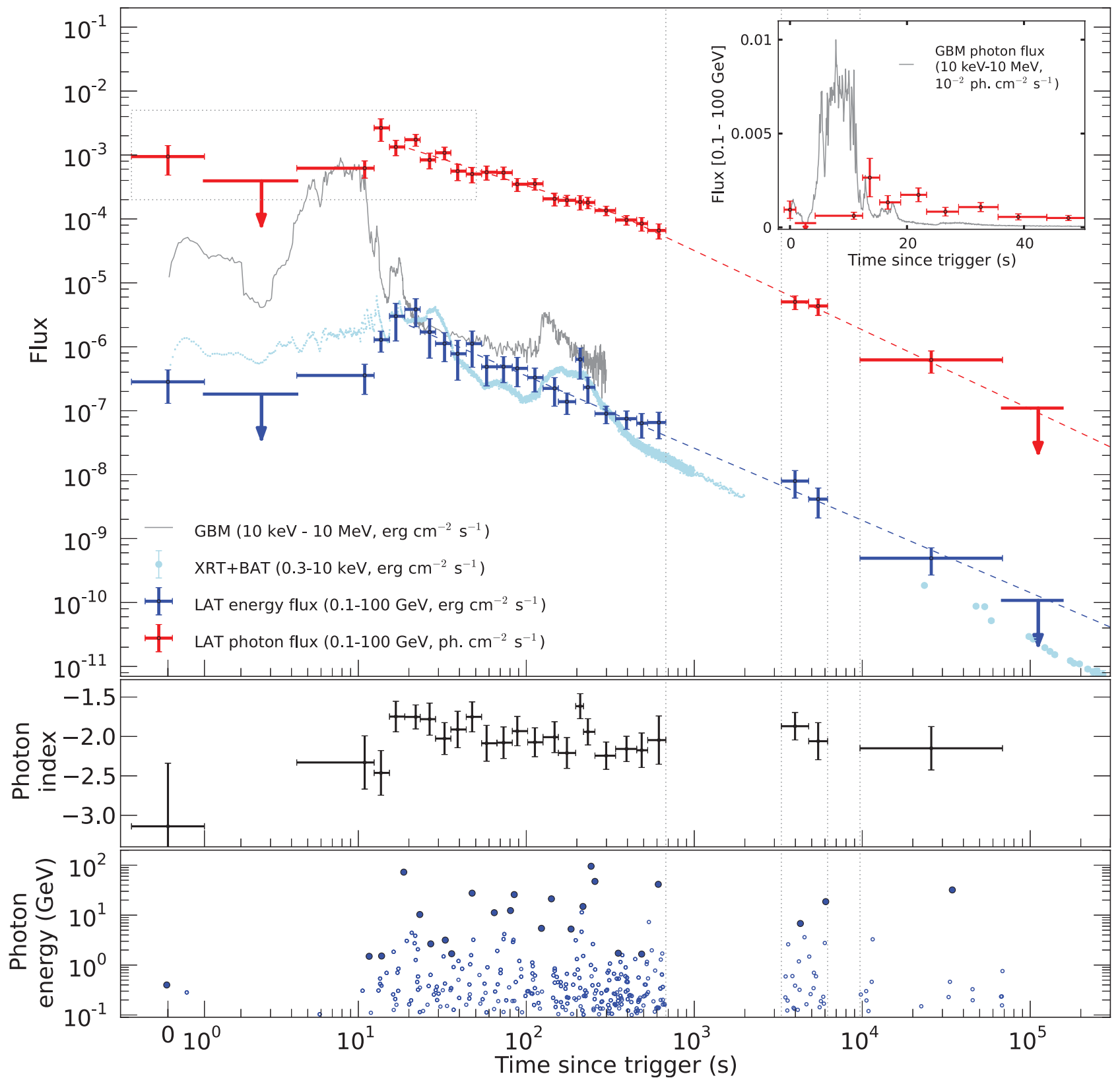

Fig. 2. Temporally extended LAT emission. Top: LAT energy flux (blue) and photon flux (red) light curves. The photon flux light curve shows a significant break at a few hundred seconds (red dashed line), whereas the energy flux light curve is well described by a single power law (blue dashed line). The $10 \mathrm{keV}$ to $10 \mathrm{MeV}$ (GBM, gray) and 0.3 to $10 \mathrm{keV}$ (XRT + BAT, light blue) energy flux light curves are overplotted. The inset shows an expanded view of the first $50 \mathrm{~s}$ with a linear axes, with the photon flux light curve from the GBM (in units of $10^{-2}$ photons $\mathrm{cm}^{-2} \mathrm{~s}^{-1}$ ) plotted in gray for comparison. Middle: LAT photon index. Bottom: Energies of all the photons with prob- abilities $>90 \%$ of being associated with the GRB (17). Solid circles correspond to the photon with the highest energy for each time interval. Note that the photons plotted here are "source" class photons, whereas the photons in Figs. 1 and 3 are "transient" class photons (17). The vertical gray lines indicate the first two time intervals during which the burst was occulted by Earth. As the autonomous repoint request moved the center of the LAT field of view toward the GRB position, the effective collecting area in that direction increased, so that after $\sim 100 \mathrm{~s}$ the rate of photons increased even though the intrinsic flux decreased. 
The explosive relativistic outflow of a GRB sweeps up and drives a shock into the circumburst medium. The medium could have, for instance, a uniform density $n_{0}\left(\mathrm{~cm}^{-3}\right)$ or a $n(r) \propto r^{-2}$ density profile resulting from the stellar wind of the type Ic supernova progenitor star associated with GRB 130427A (10). The LAT observations of GRB 130427A challenge the scenario in which the $\mathrm{GeV}$ photons are nonthermal synchrotron radiation emitted by electrons accelerated at the external forward shock $(22,23)$. In this model, the time of the brightest emission corresponds to the time $t_{\mathrm{dec}}$ when most of the outflow energy is transferred to the shocked external medium. The Lorentz factor $\Gamma\left(t_{\mathrm{dec}}\right)$ of the shock outflow in the external medium at the deceleration time $t_{\mathrm{dec}}$ is a lower limit for the initial bulk outflow Lorentz factor $\Gamma_{0}$, because a relativistic reverse shock would lower the shocked fluid Lorentz factor below $\Gamma_{0}$, and the engine time scale $T_{\mathrm{GRB}}$ can be longer than $t_{\mathrm{d}}$, the deceleration time scale for an impulsive explosion. The activity of the central engine that produces the blast wave is revealed by the $\mathrm{keV}-\mathrm{MeV}$ emission from particles accelerated at colliding-wind shocks. For GRB 130427A, the GeV emission starts to decay as a power law in time by $t \approx 20 \mathrm{~s}$ (Fig. 2), and most of the keV-MeV radiation has subsided by $t \approx 12 \mathrm{~s}$ (Fig. 1). The blast wave is in the self-similar deceleration phase at $t>t_{\mathrm{dec}}=\max \left[t_{\mathrm{d}}, T_{\mathrm{GRB}}\right]$, where $T_{\mathrm{GRB}}$ is the engine time scale (over which most of the outflow energy was released). Here, $t_{\mathrm{d}}(\mathrm{s}) \approx 2.4\left[\left(E_{\gamma, \text { iso }} / 10^{54} \mathrm{erg}\right) / n_{0}\right]^{1 / 3} /\left(\Gamma_{0} / 1000\right)^{8 / 3}$ for a uniform external medium, and $t_{\mathrm{d}}(\mathrm{s}) \approx 6.3\left(E_{\gamma, \text { iso }} /\right.$ $\left.10^{55} \mathrm{erg}\right)\left(0.1 / A_{*}\right)\left(500 / \Gamma_{0}\right)^{4}$ for a stellar wind medium of density $\rho=A R^{-2}$, with $A^{*}=A / 5 \times 10^{11} \mathrm{~g} \mathrm{~cm}^{-1}$.

Most of the fluence from GRB 130427A was radiated before $t \approx 12 \mathrm{~s}$, which suggests that $t_{\mathrm{d}} \lesssim 12$ to $15 \mathrm{~s}$. Defining $t_{1}=t_{\mathrm{d}}(10 \mathrm{~s})$ yields $t_{1} \approx 1$ to 2 , which gives $\Gamma\left(t_{\mathrm{dec}}\right) \approx 540\left[E_{55} / t_{1}^{3} n_{0}\left(\mathrm{~cm}^{-3}\right)\right]^{1 / 8}$ for the uniform-density case, where $E_{55}=E_{\gamma, \text { iso }} /\left(10^{55} \mathrm{erg}\right)$ is the isotropic energy release of the GRB. For a wind medium, $\Gamma\left(t_{\mathrm{dec}}\right) \approx 450\left\{E_{55} /\left[(A * / 0.1) t_{1}\right]\right\}^{1 / 4}$. Both values are close to the $\gamma \gamma$ opacity estimate of $\Gamma_{\min }$.

The presence of high-energy photons at times $t \gg t_{\text {dec }}$ (table S2) is incompatible with these $\gamma$ rays having a synchrotron origin. Equating the electron energy loss time scale due to synchrotron radiation with the Larmor time scale for an electron to execute a gyration gives a conservative limit on the maximum synchrotron photon energy $E_{\text {max,syn }} \approx 2^{3 / 2}\left[27 /\left(16 \pi \alpha_{\mathrm{f}}\right)\right] m_{\mathrm{e}} c^{2} \Gamma(t) /(1+z) \approx$ $79[\Gamma(t)] \mathrm{MeV}$, where $\alpha_{\mathrm{f}}$ is the fine-structure constant (17). Using $\Gamma(t)$ derived by Blandford and McKee (24) in the adiabatic limit, we find that the maximum synchrotron photon energy $E_{\text {max,syn }}<<7\left(E_{55} / n_{0}\right)^{1 / 8}[t / 200 \mathrm{~s}]^{-3 / 8} \mathrm{GeV}$, which agrees with results from integration over surfaces of equal arrival time in the self-similar regime
(25), when a scaling factor of $27 / 16 \pi$ is included (17). The presence of a $95-\mathrm{GeV}$ photon at $T_{0}+244 \mathrm{~s}$ (Fig. 4 and table S2) is incompatible with a synchrotron origin even for conservative assumptions about Fermi acceleration. This conclusion holds for adiabatic and radiative external shocks in both uniform or wind media [see also (20)]. The question of a wind or uniform-density model is not settled, but combined forward and reverse shock blast-wave model fits to the radio through x-ray emission from 0.67 days to 9.7 days after the GRB favor a wind medium (27), whereas inferences from Swift and LAT data suggest a uniform environment around GRB 130427A (7). Even in the extreme case where acceleration is assumed to operate on a time scale shorter than the Larmor time scale by a factor of $2 \pi$, synchrotron radiation cannot account for the presence of high-energy radiation in the afterglow. Synchrotron emission above $\sim 100 \mathrm{GeV}$ is still possible, however, if an acceleration mechanism faster than the Fermi process is acting, such as magnetic reconnection [e.g., (28)].

The $95-\mathrm{GeV}$ photon in the early afterglow and the $32-\mathrm{GeV}$ photon at $T_{0}+34.4 \mathrm{ks}$ therefore cannot originate from lepton synchrotron radiation in the standard afterglow model with shock Fermi acceleration (Fig. 4). If the emission mechanism for the $\mathrm{GeV}$ photons is not synchrotron radiation, the highest-energy photons can still be
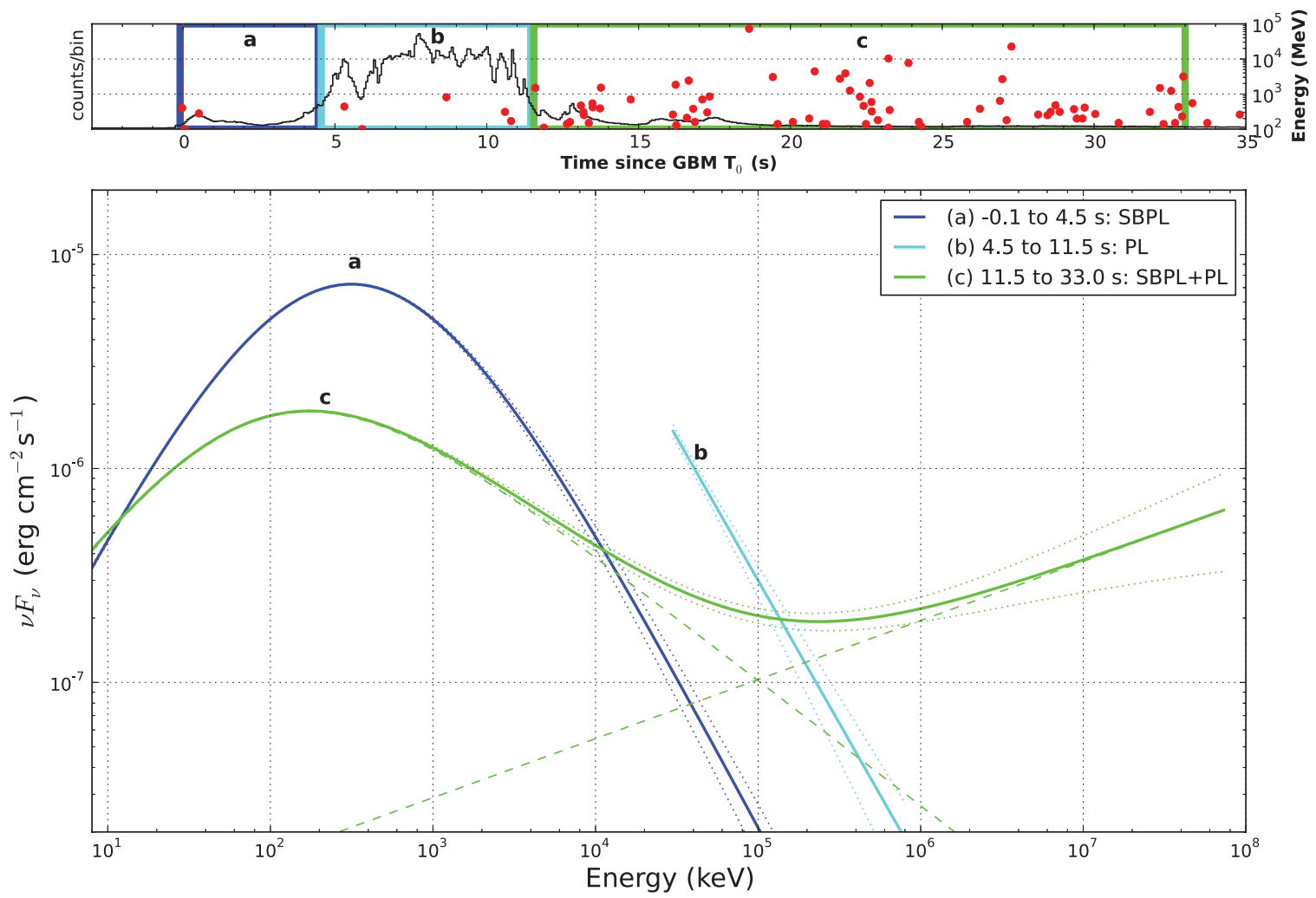

Fig. 3. Time-resolved spectral models for the GBM- and LAT-detected emission. Top: The combined Nal and BGO light curve from Fig. 1 (arbitrarily scaled) with the LAT-detected photons overplotted (same as the solid circles in Fig. 1). The time intervals are colored to correspond with the spectral models in the lower plot. The GBM data between 4.5 and $11.5 \mathrm{~s}$ are not included because they are substantially affected by pulse pile-up (17). Bottom: The models (thick lines) that best fit the data are plotted with $1 \sigma$ error contours (thin dashed lines). Each curve ends at the energy of the highest-energy LAT photon detected within that time interval. An extra power law is statistically significant when fitting the data from $T_{0}+11.5 \mathrm{~s}$ to $T_{0}+33.0 \mathrm{~s}$ (17). 
produced by lepton Compton processes. Synchrotron self-Compton (SSC) $\gamma$-rays, made when target synchrotron photons are Compton-scattered by the same jet electrons that emit the synchrotron emission, are unavoidable. SSC emission is expected to peak at $\mathrm{TeV}$ and higher energies during the prompt phase (although no GRB has been detected at $\mathrm{TeV}$ energies) and would cause the GeV light curve to flatten and the LAT spectrum to harden when the peak of the SSC component passes through the LAT waveband (29-31). Such a feature may be seen in the light curves of GRB 090902B and GRB 090926A at 15 to $30 \mathrm{~s}$ after $T_{0}(13)$, but no such hardening or plateau associated with the SSC component is observed in the LAT light curve of GRB 130427A, although extreme parameters might still allow an SSC interpretation [see, e.g., (32)]. Except for the hard flare at $t \approx 250 \mathrm{~s}$ and a possible softening at $\sim 3000 \mathrm{~s}$ [and therefore not associated with a probable beaming break at $t \approx 0.8$ days (7)], neither the integral photon nor energy-flux light curves in Fig. 2 show much structure or strong evidence for temporal or spectral variability from $t=20 \mathrm{~s}$ to $t=1$ day. The NuSTAR observations (33) of the late-time hard $\mathrm{x}$-ray afterglow also suggest that a single spectral component produces the emission from optical to multi-GeV energies. If this emission is indeed synchrotron radiation, then the standard afterglow shock model must be modified to account for the highest-energy photons detected by the LAT.

These considerations suggest that other extreme high-energy radiation mechanisms may be operative, such as external Compton processes. The most intense source of target photons is the powerful engine emissions, as revealed by the GBM and XRT prompt emission. A cocoon or remnant shell is also a possible source of soft photons, but unless the target photon source is extended and radiant, it would be difficult to model the nearly structureless LAT light curve over a long period of time. Given the similarity between the XRT and LAT light curves (Fig. 2), afterglow synchrotron radiation made by electrons accelerated at an external shock would also be the favored explanation for the LAT emission, but this is inconsistent with the detection of highenergy photons at late times.

The photon index of GRB 130427A, -2, is similar to those found in calculations of electromagnetic cascades created when the $\gamma$-ray opacity of ultrahigh energy (UHE, $>100 \mathrm{TeV}$ ) photons in the jet plasma is large (34). An electromagnetic cascade induced by ultrarelativistic hadrons would be confirmed by coincident detection of neutrinos, but even for GRB 130427A with its extraordinary fluence, only a marginal detection of neutrinos is expected with IceCube, and none has been reported (35). Because the UHE $\gamma$-ray photons induce cascades both inside the radiating plasma and when they travel through intergalactic space [e.g., $(36,37)]$, a leptonic or hadronic cascade component in GRBs, which is a natural extension of colliding shell and blast wave models, might be required to explain the high-energy emission of GRB 130427A provided that the required energies are not excessive. The observations described above demonstrate nonsynchrotron emission in the afterglow phase of the bright GRB 130427A, contrary to the hitherto standard model of GRB afterglows.
Fig. 4. Curves of maximum synchrotron photon energy. The black dots show the LAT detection times of photons with energies greater than $1 \mathrm{GeV}$ and $>90 \%$ probability of association with GRB 130427A. Adiabatic and radiative predictions for maximum synchrotron photon energy in uniform interstellar medium (ISM) and wind environments are plotted using the relations described in (17). Red and blue curves refer to the ISM and wind cases, respectively. The solid and dashed lines refer to the adiabatic and radiative cases with $\Gamma_{0}=1000$, and the dot-dashed and

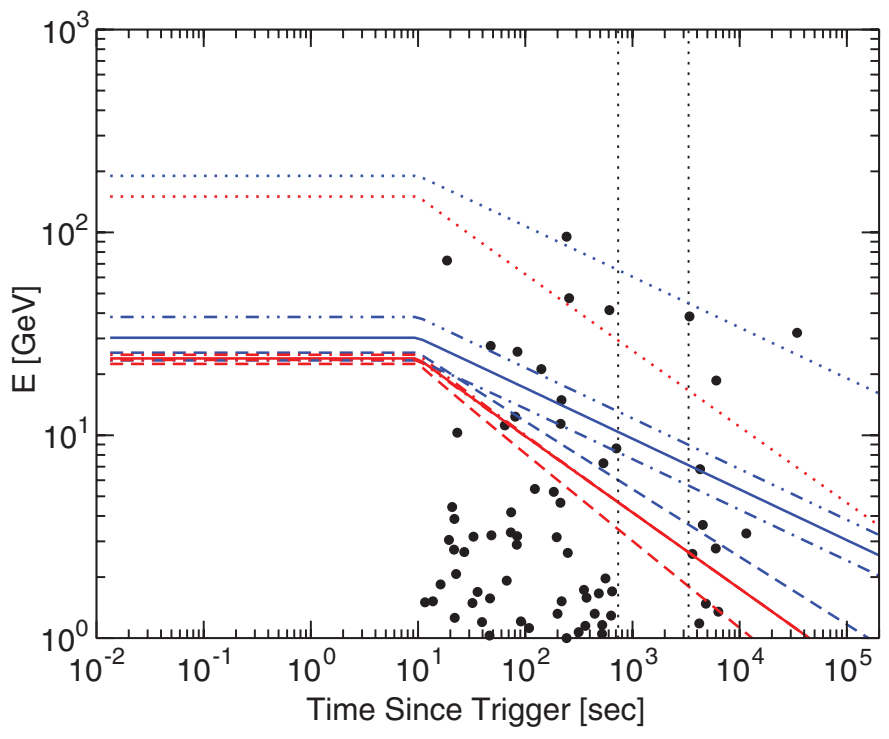

double dot-dashed lines represent the adiabatic case with $\Gamma_{0}=500$ and $\Gamma_{0}=2000$, respectively. The dotted lines show an extreme possibility where acceleration takes place on the inverse of the Larmor angular frequency, in the case of an adiabatic blast wave with $\Gamma_{0}=1000$. For cases with uniform external medium, $E_{\mathrm{iso}}\left(10^{55} \mathrm{erg}\right) / n_{0}\left(\mathrm{~cm}^{-3}\right)=1$. The wind normalization was chosen to give the same value of $t_{\mathrm{d}}$ for both wind and ISM cases. The vertical dotted lines show periods of Earth avoidance when the LAT could not observe GRB 130427A.
References and Notes

1. W. Atwood et al., Astrophys. J. 697, 1071-1102 (2009)

2. C. Meegan et al., Astrophys. J. 702, 791-804 (2009).

3. N. Gehrels, S. Razzaque, Front. Phys. 10.1007/ s11467-013-0282-3 (2013).

4. A. Levan et al., GCN Circ. 14455 (2013).

5. K. Hurley et al., Nature 372, 652-654 (1994).

6. A. von Kienlin, GCN Circ. 14473 (2013).

7. A. Maselli et al., Science 343, 48-51 (2014).

8. D. Perley, GCN Circ. 14494 (2013).

9. W. T. Vestrand et al., Science 343, 38-41 (2014).

10. D. Xu et al., http://arxiv.org/abs/1305.6832 (2013).

11. A. Abdo et al., Astrophys. J. 706, L138-L144 (2009).

12. LLE is a set of cuts that provides increased statistics at the cost of a higher background level and greatly reduced energy and angular reconstruction accuracy.

13. M. Ackermann et al., http://arxiv.org/abs/1303.2908 (2013).

14. V. Pelassa et al., http://arxiv.org/abs/1002.2617 (2010).

15. D. Band et al., Astrophys. ]. 413, 281 (1993).

16. A. Goldstein et al., Astrophys. J. Suppl. Ser. 199, 19 (2012).

17. See supplementary materials on Science Online.

18. P.-H. T. Tam, Q.-W. Tang, S.-J. Hou, R.-Y. Liu, X.-Y. Wang, Astrophys. J. 771, L13 (2013).

19. S. B. Cenko et al., Astrophys. J. 732, 29 (2011).

20. P. Guilbert et al., Mon. Not. R. Astron. Soc. 205, 593 (1983).

21. ]. Granot, ]. Cohen-Tanugi, E. do Couto e Silva, Astrophys. J. 677, 92-126 (2008).

22. P. Kumar, R. Barniol-Duran, Mon. Not. R. Astron. Soc. 400, L75-L79 (2009)

23. G. Ghisellini, G. Ghirlanda, L. Nava, A. Celotti, Mon. Not. R. Astron. Soc. 403, 926-937 (2010).

24. R. Blandford, C. McKee, Phys. Fluids 19, 1130 (1976).

25. T. Piran, E. Nakar, Astrophys. J. 718, L63-L67 (2010).

26. Y.-Z. Fan et al., http://arxiv.org/abs/1305.1261 (2013).

27. T. Laskar et al., http://arxiv.org/abs/1305.2453 (2013)

28. D. Giannios, Astron. Astrophys. 480, 305-312 (2008).

29. C. Dermer, J. Chiang, K. E. Mitman, Astrophys. J. 537 , 785-795 (2000)

30. R. Sari, A. Esin, Astrophys. J. 548, 787-799 (2001)

31. B. Zhang, P. Mészáros, Astrophys. ]. 559, 110-122 (2001).

32. R.-Y. Liu et al., Astrophys. J. 773, L20 (2013).

33. C. Kouveliotou et al., Astrophys. ]. 10.1088/2041-8205/ 779/1/L1 (2013).

34. C. Dermer, A. Atoyan, New J. Phys. 8, 122 (2006).

35. E. Blaufuss, GCN Circ. 14520 (2013).

36. S. Razzaque, P. Meszaros, B. Zhang, Astrophys. J. 613 1072-1078 (2004)

37. K. Murase, Astrophys. J. 745, L16 (2012).

Acknowledgments: The Fermi LAT Collaboration acknowledges support from a number of agencies and institutes for both development and the operation of the LAT as well as scientific data analysis. These include NASA and DOE in the United States; CEA/Irfu and IN2P3/CNRS in France; ASI and INFN in Italy; MEXT, KEK, and JAXA in Japan; and the K. A. Wallenberg Foundation, the Swedish Research Council, and the National Space Board in Sweden. Additional support from INAF in Italy and CNES in France for science analysis during the operations phase is also gratefully acknowledged. The Fermi GBM collaboration acknowledges support for GBM development, operations and data analysis from NASA in the United States and BMWi/DLR in Germany.

\section{Supplementary Materials}

www.sciencemag.org/content/343/6166/42/suppl/DC1

Materials and Methods

Figs. S1 and S2

Tables S1 and S2

References (38-50)

24 June 2013; accepted 21 October 2013

Published online 21 November 2013;

$10.1126 /$ science. 1242353 
DOI: $10.1126 /$ science. 1242353

This copy is for your personal, non-commercial use only.

If you wish to distribute this article to others, you can order high-quality copies for your colleagues, clients, or customers by clicking here.

Permission to republish or repurpose articles or portions of articles can be obtained by following the guidelines here.

The following resources related to this article are available online at www.sciencemag.org (this information is current as of February 3, 2015 ):

Updated information and services, including high-resolution figures, can be found in the online version of this article at:

http://www.sciencemag.org/content/343/6166/42.full.html

Supporting Online Material can be found at:

http://www.sciencemag.org/content/suppl/2013/11/20/science.1242353.DC2.html

http://www.sciencemag.org/content/suppl/2013/11/20/science.1242353.DC1.html

A list of selected additional articles on the Science Web sites related to this article can be found at:

http://www.sciencemag.org/content/343/6166/42.full.htmI\#related

This article cites $\mathbf{3 4}$ articles, 9 of which can be accessed free:

http://www.sciencemag.org/content/343/6166/42.full.html\#ref-list-1

This article has been cited by 8 articles hosted by HighWire Press; see:

http://www.sciencemag.org/content/343/6166/42.full.html\#related-urls

This article appears in the following subject collections:

Astronomy

http://www.sciencemag.org/cgi/collection/astronomy 